\title{
Rotavirus Hospital Surveillance in the Era of Immunization
}

\author{
Aja M. Sanzone $e^{1,2}$ and Rodolfo E. Bégué ${ }^{1,2, *}$ \\ ${ }^{1}$ Louisiana State University Health Sciences Center, USA \\ ${ }^{2}$ Children's Hospital, New, Orleans, Louisiana, USA
}

\begin{abstract}
Background: In 2006 a rotavirus (RV) vaccine (Rota Teq ${ }^{\circledR}$ ) was licensed for routine childhood immunization in the US. The aim of the present study was to track changes in RV hospital visits and to monitor circulating RV genotypes as vaccination was introduced into New Orleans, Louisiana.

Methods: From July 2005 to June 2009, RV-positive stool specimens were systematically collected, viral RNA was extracted and subjected to semi-nested multiplex Reverse-Transcription PCR with primers specific for the gene $9(\mathrm{G})$ types G1, G2, G3, G4, G9, and the gene 4 (P) types P[4], P[6], P[8], P[9] and P[10]. Clinical records of RV-positive children were reviewed.

Results: As RV-vaccination was introduced, there was a decrease in the rate of RV-positive cases (per 1,000 hospital visits) from 3.82 to 0.73 . A change was noted in the prevailing genotypes, from a predominance of G1P[8] strains to a predominance of G3P[8]. Most affected children were unimmunized. Sequence analysis of gene 9 and gene 4 showed that the circulating strains remained relatively stable over the years studied in this project.

Conclusion: In New Orleans, G3P[8] is becoming a predominant strain of RV. The cause of this change observed has not been determined. It is unclear whether this phenomenon represents natural variation or it is caused by introduction of vaccines.
\end{abstract}

Keywords: Epidemiology, gastroenteritis, genotype, rotavirus, rotavirus vaccine.

\section{INTRODUCTION}

Rotaviruses (RV) represent the main cause of severe, dehydrating diarrhea in infants and young children. In the $\mathrm{US}$, in the pre-vaccine era, RV were responsible for more than 2.7 million infections, 410,000 office visits, 205,000272,000 emergency department (ED) visits, 55,000-70,000 hospitalizations and 20-60 deaths every year, translating into approximately 1 billion dollars expenses [1]. To control the burden of RV disease, two vaccines have been made available for routine childhood immunization in the US: Rota Teq $^{\circledR}$ (RV5, Merck \& Co., Inc) and Rotarix ${ }^{\circledR}$ (RV1, GlaxoSmithKline). The American Academy of Pediatrics recommends routine immunization of infants with either RV5 or RV1 [2]. Of the two, RV5 has been the vaccine predominantly utilized in New Orleans. Pre-licensure studies of RV5 showed $74 \%$ (95\% confidence interval $[\mathrm{CI}]=67 \%$, $80 \%$ ) efficacy in preventing RV-cases of any severity and $96 \%$ $(95 \% \mathrm{CI}=91 \%, 98 \%)$ to prevent hospitalizations [1,3]. RV5 was licensed in 2006 and, soon after, the Centers for Disease Control and Prevention (CDC, Atlanta, GA) reported that for the 2007-08 season there was a decrease and delay in the regular RV yearly winter season, concomitant with the increased use of the vaccine nation-wide [4]. This decrease

*Address correspondence to this author at the Rodolfo E Bégué, MD, Children's Hospital, 200 Henry Clay Avenue, New Orleans, LA 70118, USA; Tel: (504) 896-9820; Fax: (504) 894-5137; E-mail: rbegue@lsuhsc.edu in RV-related hospitalization was confirmed by a recent publication from the Children's Hospital of Philadelphia [5].

Typing of RV strains is based on the two outer capsid proteins: viral protein 7 (VP7) and VP4 [6]. VP7 is encoded in gene 9 and the various types are called G types. There are at least 19 described $\mathrm{G}$ types with 4 of them (G1-G4) being the most common in the US and accounting for $90 \%$ of isolates [1]. Unusual G types (such as G9, G12 and others) are being increasingly recognized in the US $[7,8]$. VP4 is encoded in gene 4 and the various genotypes are called $P$ types and indicated in brackets. There are at least $28 \mathrm{P}$ types described; the most common is $\mathrm{P}[8]$ which accounts for $85 \%$ isolates in the US [1]. RV strains are designated by a binary system of $G$ and $P$ specificity. Overall, the most common strains are G1P[8], accounting for $78 \%$ isolates in the US [1], but there is marked natural year-to-year variation in the prevailing types [9]. RV5 has been formulated to contain human-bovine RV reassortant strains representative of the most common G and P types, ie, G1, G2, G3, G4 and P[8]. However, since not all types are represented, it is possible that the immunologic pressure introduced by vaccination may lead to the emergence of uncommon genotypes, including some not included in the current vaccines.

The aim of the present study was to track changes in RV hospital visits (either ED visits or hospital admissions) as RV vaccines are introduced in our community and to monitor changes in the circulating RV genotypes. These data 
would have potential public health implications regarding the impact of RV immunization on the disease.

\section{METHODS}

\section{Surveillance Site}

Children's Hospital, New Orleans, a 200-bed facility, is the main tertiary care pediatric center in the area, attending approximately 45,000 annual ED visits. Although at the discretion of the attending physician, it is routine - especially during the winter season - to test a stool specimen of any child presenting with diarrhea by the use of a commercially available enzyme immune assay (Rotaclone, Meridian Diagnostics) for the possibility of RV etiology. From July 2005 to June 2009, specimens that tested positive for RV were systematically stored at $-70{ }^{\circ} \mathrm{C}$ until processed for genotyping. Data from the 5 years previous to the study showed that rotavirus season last 3-4 months in New Orleans with peaks fluctuating between January and April. For the purpose of this study, each surveillance season spanned from July $1^{\text {st }}$ of a given year to June $30^{\text {th }}$ the following year. Since we could not directly measure the amount of $\mathrm{RV}$ vaccine used in the community, we used the data of a sentinel pediatric practice, the Children's Hospital Medical Practice Corporation (CHMPC) which represents the largest practice referring patients to Children's Hospital, New Orleans, to estimate the approximate level of vaccine utilization (data kindly provided by Dr. Keith Perrin). Vaccine utilization was calculated as the percent of children $<1$ year of age seen at the practice in a given surveillance season that received at least 1 dose of RV5. This sentinel pediatric practice has used RV5 exclusively as their RV vaccine since mid-2006.

\section{Genotyping}

The protocol utilized by the CDC and kindly provided by Dr. Jon Gentsch was used for genotyping [10]. Briefly, stool specimens were thawed, a $1: 5$ to $1: 10$ suspension in RNase/DNase-free phosphate buffered saline (either weight/ $\mathrm{vol}$ or vol/vol, as appropriate) was prepared, viral RNA was extracted (using QIAamp Viral RNA Kit, Qiagen) and subjected to semi-nested multiplex Reverse-Transcription PCR (iCycler Thermal Cycler, Bio-Rad Laboratories, Hercules, CA, USA). The first round of amplification used Qiagen's One Step RT-PCR kit for reverse-transcription and amplification with primers that targeted consensus sequences of either gene 9 or gene 4 of RV. G typing primers 9 con $1 \mathrm{~L}$ and VP7R (bp: 37-933) and P typing primers con3 and con2 (bp: 11-877) were used. Regular PCR amplification (anneal at 42 ${ }^{\circ} \mathrm{C}$ ) was used for the second round utilizing the same consensus 5'-primers as the first round mixed with a pool of $\mathrm{G}$ or $\mathrm{P}$ type specific 3'-primers: G typing primers G1 (9T-1, 9T1DG), G2 (9T-2), G3 (93-TP), G4 (9T-4), G9 (9T-9B) and P typing primers $\mathrm{P}[4]$ (2T-1), $\mathrm{P}[6]$ (3T-1), $\mathrm{P}[8]$ (1T-1, 1T1Vietnam, JRG237), P[9] (4T-1) and P[10] (5T-1). Each G or $P$ type is identified based on its corresponding and different sized amplicon. The sequences for the primers and amplicon sizes are specified in the reference [10]. Selected G and P first round amplicons were sent to Louisiana State University Health Sciences Center, Genomics Core Facility, for sequencing.

\section{Clinical Information}

Clinical records (either ED or admit) of children identified with RV infection were reviewed to determine age, need for admission and length of stay (as surrogates for disease severity). Hospital-acquired infections (i.e, those evident $>3$ days after admission) were excluded from the study. CHMPC records were reviewed to obtain information on receipt of RV vaccine by the child. Additional baseline (pre-vaccine) data from season 2004-05 was collected.

\section{Human Subject Research}

Protocol review, approval and oversight were provided by the Institutional Review Board of Louisiana State University Health Sciences Center and Children's Hospital, New Orleans, LA. The study was granted Waiver of HIPAA Authorization and Waiver of Informed Consent status.

\section{Statistical Analysis}

For statistical analyses, Epi-Info's Statcalc program (EpiInfo, version 3.5, CDC, Atlanta, GA) was used to calculate ratios and $95 \%$ Confidence Intervals $(95 \% \mathrm{CI})$. Proportions were compared by calculation of 2-sided chi-square test with Yates correction.

\section{RESULTS}

\section{Rotavirus Activity (Table 1)}

Before the introduction of RV vaccination (season 200405 and 2005-06), the rate of RV-positive cases per 1,000 ED visits remained relatively constant at 3.82 and 3.79 , respectively. In 2006-07, the year RV5 was introduced in the community (in the sentinel practice the utilization was $18 \%$ ), there was a modest but significant decrease (28\% decrease, $95 \% \mathrm{CI}=9 \%, 42 \%$ ) in the rate of RV-positive cases to 2.76 , with further decrease to $0.73(81 \%$ decrease, $95 \% \mathrm{CI}=73 \%$, $87 \%$ ) in 2007-08 when RV5 was more widely utilized $(50 \%$ in sentinel practice). In 08-09, despite a continue use of RV5 (56\% in the sentinel practice) an increase to 1.55 was noted in the rate of RV activity detected. This rate of RV disease for 2008-09 was significantly higher than the previous 200708 season $(113 \%$ increase, $95 \% \mathrm{CI}=43 \%, 217 \%)$; the overall rate of RV disease, however, still remained lower $(59 \%$ decrease, $95 \% \mathrm{CI}=47 \%, 69 \%$ ) than pre-vaccine rates.

\section{Clinical Characteristics (Table 2)}

During the first 4 seasons under study, two of which preceded the introduction of the vaccine (2004-05 and 2005-06) and two of which were subsequent to the introduction of the vaccine (2006-07 and 2007-08), the age of the RV-positive patients remained stable (mean $=2.3$ years) with $20.2 \%(94$ of 465) of these children being 3 years and older. In contrast, during the last season (2008-09), the age of the affected patients increased (mean $=2.9$ years) with $37.8 \%$ of them $(30$ of 79; $\mathrm{p}<0.0001$ ) being 3 years and older. It should be noted that children 3 years and older were too old to have received the vaccine in the years under study. The proportion of RVpositive patients hospitalized (77\%) and their length of stay (mean $=2.3$ days) remained constant over the entire study period. 
Table 1. Percent of Children $<1$ Year of Age Vaccinated with $\geq 1$ Dose of Rotavirus Vaccine at the Sentinel Practice and Rate (per 1,000 Emergency Department Visits) of Rotavirus-Positive Cases Identified at Children's Hospital of New Orleans

\begin{tabular}{|c|c|c|c|c|c|}
\hline & \multicolumn{4}{|c|}{ Surveillance Season } \\
\cline { 2 - 6 } & $\mathbf{2 0 0 4 - 0 5}$ & $\mathbf{2 0 0 5 - 0 6}$ & $\mathbf{2 0 0 6 - 0 7}$ & $\mathbf{2 0 0 7 - 0 8}$ & $\mathbf{2 0 0 8 - 0 9}$ \\
\hline \hline RV vaccine use (\%) & 0 & 0 & 18.4 & 50.2 & 47,950 \\
\hline No. ED visits & 50,575 & 29,817 & 44,947 & 36.2 & 50,952 \\
\hline No. RV-positive & 193 & 113 & 124 & 0.73 & 79 \\
\hline Rate $^{\text {a }}$ & 3.82 & 3.79 & 2.76 & 1.55 \\
\hline
\end{tabular}

${ }^{\text {a }}$ Rate per 1,000 ED visits; ED = Emergency Department; RV = rotavirus.

Table 2. Clinical Characteristics of Rotavirus-Positive Cases Seen at Children's Hospital, New Orleans

\begin{tabular}{|c|c|c|c|c|c|}
\hline & \multicolumn{4}{|c|}{ Surveillance Season } \\
\cline { 2 - 6 } & $\mathbf{2 0 0 4 - 0 5}$ & $\mathbf{2 0 0 5 - 0 6}$ & $\mathbf{2 0 0 6 - 0 7}$ & $\mathbf{2 0 0 7 - 0 8}$ & $\mathbf{2 0 0 8 - 0 9}$ \\
\hline \hline No. cases & 193 & 113 & 124 & 35 \\
\hline Age (years) & a & $1.97(2.14)$ & $2.48(3.07)$ & $2.29(2.74)$ & $2.00(1.61)$ \\
$5(14.3)$ & $28(22.6)$ & $2.90(2.62)$ \\
No. (\%) $\geq 3$ years & $34(17.6)$ & $27(23.9)$ & $98(79.0)$ & $21(60.0)$ & $55(69.7)$ \\
No. (\%) admitted & $152(78.8)$ & $94(83.2)$ & $2.73(2.77)$ & $2.19(0.98)$ \\
\hline Length of stay (days)
\end{tabular}

${ }^{\text {a }}$ mean (standard deviation).

\section{Immunization Status}

RV5 was made available to the area in August-2006. Of the 114 children detected with RV-infection during the 200708 and 2008-09 seasons, 43 (38\%) were CHMPC patients. Of them, 20 were out of the age range indicated for RV5 immunization (either too young or too old) and of the 23 age-eligible children (mean age: 1.6 years; $\mathrm{SD}=0.58$ years), only $2(8.7 \%)$ had received RV5. Neither of these 2 children received the complete 3 -dose vaccine series. One child received a single vaccine dose (RV infection 6 months post vaccination) and the other child received 2 doses of the vaccine (RV infection 16 months post vaccination). The genotypes detected in these two partially vaccinated children were $\mathrm{G} 1 \mathrm{P}[8]$ and $\mathrm{G} 3 \mathrm{P}[8]$, respectively. In contrast, 1165 children (totaling 1,469 episodes) with RV-negative diarrhea were detected during the same period; of them, $253(22 \%)$ were CHMPC patients, and $43(30 \%)$ of the 142 age-eligible children (mean age: 0.92 years; $\mathrm{SD}=0.55$ years) had received at least one dose RV5 (18 one dose, 24 two doses and 18 three doses) before the episode of diarrhea. Hence, having received RV5 was more common among RVnegative than RV-positive cases $(p=0.031)$.

\section{Genotype Distribution (Table 3)}

Throughout seasons 2005-06 to 2008-09, a total of 351 RV-positive patients were identified and 221 (63\%) had specimens available for genotyping. Four specimens could not be typed (no first- or second-round PCR product obtained despite multiple attempts with the consensus and type specific primers). In the 2005-06 season (pre-vaccine), G1P[8] was the predominant genotype accounting for $78 \%$ of the typed specimens, and G2P[4] was second most common accounting for $14 \%$ of the total typed specimens. In $2006-07$, as the vaccine was introduced in the community (18\% vaccine utilization), G1P[8] genotype decreased (42\% of cases), while G2P[4] increased (25\% of cases) and a wide variety of other genotypes appeared including G3P[8] which accounted for $12 \%$ of cases. In 2007-08 (vaccine utilization $50 \%$ ), a significant decrease in the number of specimens was noted with equal representation of G1P[8] and G3P [8] (about $50 \%$ of cases, each). Finally, in 2008-09, (vaccine utilization $56 \%$ ) G3P[8] became the predominant genotype (85\% of cases) and G1P[8] fell to the second most common type (12\% of cases).

\section{G3 and P[8] Sequences (Table 4)}

Since the last season was characterized by an increase in infections by G3P[8] genotypes despite those 2 types being present in RV5, representative strains from each season (except season 2004-05 which could not be amplified) were sequenced and compared to the respective strains present in RV5. These were partial sequences of either the gene 9 or gene 4 first round amplicon $(\sim 750 \mathrm{bp})$. To assign the nucleotide and amino acid positions, reference sequences (GenBank accession number EU159191 for VP7 and L34161 for 
Table 3. Rotavirus Genotype Distribution Over the Years

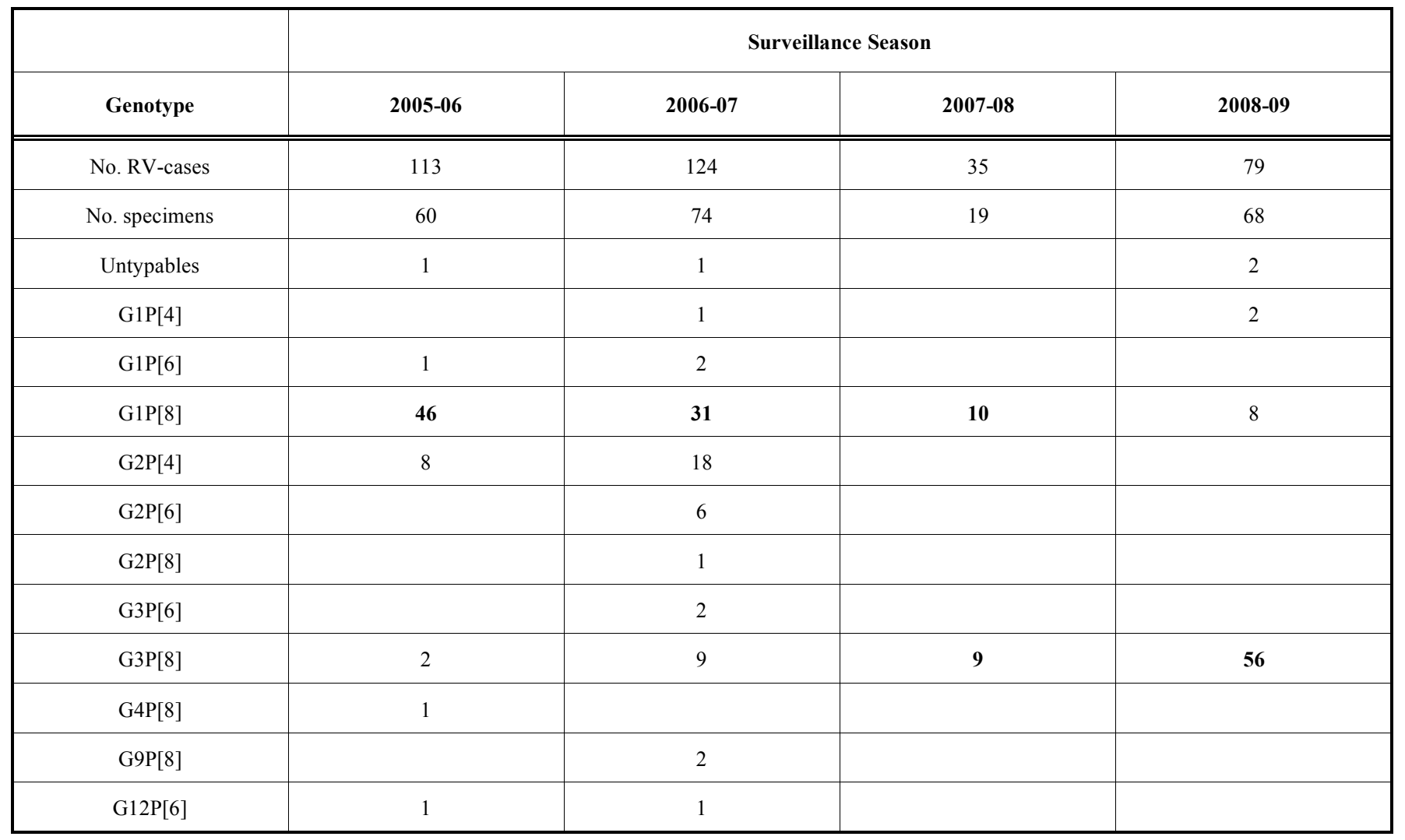

Note: Predominant genotypes are highlighted in bold.

VP4) were used. G3 sequences were 93.6\%-94.2\% homologous to RV5's G3 strain at the nucleotide level and $96.4 \%$ $97.2 \%$ at the amino acid level. P[8] strains had few more amino acid changes compared to RV5's strain, with homology of $92.7 \%-93.4 \%$ at the nucleotide level and $93.7 \%$ $94.9 \%$ at the amino acid level. Both G3 and P[8] strains proved to be very stable over time with very few additional amino acid changes noted.

\section{DISCUSSION}

The goal inherent in the introduction of each vaccine is to reduce the burden of the disease targeted in the appropriate population. In that regard, similar to what has been described by others, our study found that concomitant with the introduction of RV5 in the community there was a decrease in the rate of RV-disease [4, 5]. Interestingly, in 2008-09, despite continue use of the vaccine, we noted an increase in the number of cases of RV, even though the rate remained about $50 \%$ lower than the corresponding value before vaccination. This increased detection did not seem to be caused by a change in testing practices since we found that the rate of RV testing (per 1,000 ER visits) at Children's Hospital remained constant over the years studied (mean $=18.0$; annual range: $16.5-19.5)$.

But as the pathogen ceases to circulate, an ecological void is created that could be filled by related agents. For example, introduction of the pentavalent pneumococcal vaccine has been associated with a marked decrease in disease caused by Streptococcus pneumoniae [11], yet the benefit has been partially offset by an increase in the infection caused by serotypes not included in the vaccine, particularly serotype 19A [12-14]. Whether a similar phenomenon will occur with introduction of the RV vaccines must be investigated. In our study we saw a decrease in prevalence of the common G1P[8] genotypes and an increase in the uncommon G3P[8] genotype. This finding is similar to what has been recently described at Children's Hospital of Philadelphia [5]. RV is a segmented virus; like influenza it reassorts easily. As a result, RV genotype variations occur frequently and seasons marked by unusual genotypes have been previously documented [7]. Predominance of G3P[8] isolates happen to be uncommon, though. We do not have preexisting data on RV genotypes in New Orleans, but in a study in Southern New England, G3 strains (by serotyping and RNA electrophoresis) were predominant in only 1 of 9 seasons (1982-91) investigated [9], and in Philadelphia the previously G3-predominant season occurred 13 years (199495) before their current emergence of G3P [8] genotypes [5]. Furthermore, a recent comprehensive review of RV genotypes circulating in several cities from different U.S. regions found only 54 of $3,159(1.7 \%)$ isolates to be G3P[8], and this genotype did not predominate in any of the seasons studied (1996-97 through 2004-05) [15]. So, even though the present G3P [8] predominance may be explained by natural variation, that seems an unusual event. The role that the vaccine may be playing in this shift of genotypes is uncertain and will require additional years of surveillance. 
Table 4. G3 and P[8] Sequences of Selected Strains Over the Years, as Compared to RV5's (Rota Teq). To Determine Amino Acid Positions, GenBank Sequences (EU159191 and L34161 for G3 and P[8], Respectively) were used. Footnote Indicates Amino Acid Substitution, as Compared to RV5

\section{G3 Sequences}

\begin{tabular}{|c|c|c|c|c|c|c|c|c|c|c|c|c|}
\hline \multirow{2}{*}{$\frac{\text { Year }}{2006-07}$} & \multirow{2}{*}{$\begin{array}{c}\text { Strain } \\
0607\end{array}$} & \multicolumn{11}{|c|}{ Amino Acid Differences as Compared to RV5 Sequence } \\
\hline & & 65 & & & 107 & 113 & 211 & & & 237 & 241 & 265 \\
\hline & 0618 & 65 & & & 107 & 113 & 211 & & & 237 & 241 & 265 \\
\hline & 0625 & 65 & & & 107 & 113 & 211 & & & 237 & 241 & 265 \\
\hline \multirow[t]{2}{*}{ 2007-08 } & 0711 & 65 & & & 107 & 113 & 211 & & 217 & 237 & 241 & \\
\hline & 0718 & 65 & & & 107 & 113 & 211 & & 217 & 237 & 241 & \\
\hline \multirow[t]{6}{*}{ 2008-09 } & 0802 & 65 & & & 107 & 113 & 211 & & 217 & 237 & 241 & \\
\hline & 0817 & 65 & 71 & & 107 & 113 & 211 & & 217 & 237 & 241 & \\
\hline & 0828 & 65 & 71 & & 107 & 113 & 211 & & 217 & 237 & 241 & \\
\hline & 0841 & 65 & & & 107 & 113 & 211 & 212 & 217 & 237 & 241 & \\
\hline & 0851 & 65 & 71 & 99 & 107 & 113 & 211 & & 217 & 237 & 241 & \\
\hline & 0861 & 65 & & & 107 & 113 & 211 & & & 237 & 241 & \\
\hline
\end{tabular}

S65P, R71Q, D99N, T107I, E113G, A211T, N212S, V217I, K237N, D241N, S265P.

\section{$P[8]$ Sequences}

\begin{tabular}{|c|c|c|c|c|c|c|c|c|c|c|c|c|c|c|c|c|c|c|}
\hline$\frac{\text { Year }}{2006-07}$ & $\begin{array}{c}\text { Strain } \\
0607\end{array}$ & 51 & 77 & 105 & 107 & & 113 & 119 & & 146 & 149 & 161 & 172 & 194 & 198 & & 241 & 251 \\
\hline & 0625 & 51 & 77 & 105 & 107 & & 113 & 119 & & 146 & 149 & 161 & 172 & 194 & 198 & & 241 & 251 \\
\hline \multirow[t]{4}{*}{ 2008-09 } & 0802 & 51 & 77 & 105 & 107 & 112 & & 119 & 144 & & 149 & 161 & & 194 & 198 & & & 251 \\
\hline & 0817 & 51 & 77 & 105 & 107 & 112 & & 119 & 144 & & 149 & 161 & & 194 & 198 & & & 251 \\
\hline & 0828 & 51 & 77 & 105 & 107 & 112 & & 119 & 144 & & 149 & 161 & & 194 & 198 & & & 251 \\
\hline & 0841 & 51 & 77 & 105 & 107 & 112 & & 119 & 144 & & 149 & 161 & & 194 & 198 & & & 251 \\
\hline
\end{tabular}

Y51H,I77T, V105I, I107V, N112D, P113S, T119N, S144G, S146N, E149D, K161R, I172V, D194G, I198T, S212F, I241V, E251D.

Reassuringly, the vaccine appears to remain protective to the currently circulating strains, as only 2 cases of RV disease in partially immunized children were detected in our study. In line with the finding that the vaccine remains effective is the fact that the now circulating G3P[8] strains in our area remain relatively stable at the gene level (at least for the genes and segments sequenced). Some of the amino acid differences detected fall within regions of VP7 (amino acid 87-101 and 208-221) [16-19] and VP4 (amino acid 180, 183, 228-241) $[20,21]$ that have been suggested as important for immune protection. Yet the circulating strains do not seem to be evading vaccine-induced immunity. Another finding of our study is that the age of children affected by RV disease is increasing, even though the severity of the disease - at least for those requiring an ED visit - remains the same. In other words, the prevailing G3P[8] genotype seems to be affecting either young unimmunized children, or older children with no natural immunity to this strain, which likely has only rarely circulated in the community for the past few years. 
Few limitations to our study should be noted. First, our study looks at the short-term phenomena of introduction of $\mathrm{RV}$ vaccination and changes in the epidemiology of the disease; the link between the two and possible causality will become more clear with ongoing observation. It is likely that the scenario will continue to evolve as either natural or vaccine-induced immunity changes in the community. G3P[8] may remain predominant or it may be replaced by other types and the clinical presentation of RV-disease may change as well. Second, we have limited data from pre-vaccine years since we only recently initiated our project, so we must rely on regional and national data to help interpret our findings. Third, our data comes from a single hospital facility in New Orleans. Still, Children's Hospital represents the largest pediatric facility in the area accounting for about $50 \%$ pediatric hospitalizations in New Orleans and hence - even though not complete - it likely provides a good indication of RV disease severe enough to be seen in the ED or hospitalized. Fourth, since we did not have access to community-wide information on RV vaccination, we used a sentinel practice to monitor this parameter. The sentinel practice is a group of 38 physicians practicing in 14 locations across the Greater New Orleans area with a large patient base representative of the community and accounts for $18.5 \%$ of patients seen for acute gastroenteritis at Children's Hospital. We don't know how well the use of RV vaccine in this practice reflected the use in other practices but this was the best source of information that we had available to the study. Fifth, there was no systematic RV testing of all children with diarrhea but rather that was left to the discretion of the attending practitioner. Still, it is common practice at Children's Hospital to test most children with diarrhea for RV, especially during the winter. For example, for CHMPC hospitalized patients we found that most cases were tested for RV etiology (81\%) and that number remained constant over the study years (range: $81-82 \%$ ). Finally, RV severity was defined by hospitalization and length of stay. A standard severity score (like the 20-point Vesikari scale) likely would have been more sensitivity to detect changes in severity over the years, but we encountered much missing data, making its use unreliable.

In summary, our study shows that in the immediate period following introduction of RV vaccine in our area, the epidemiology of the disease and the genotype distribution of the prevailing strains seem to be changing. Understanding the relationship between these events as well as the direction that these changes will undertake in the future will require ongoing surveillance.

\section{FINANCIAL SUPPORT}

Supported in part by a research grant from the Investigator-Initiated Studies Program of Merck \& Co., Inc. The opinions expressed in this paper are those of the authors ad do not necessarily represent those of Merck \& Co., Inc.

\section{CONFLICT OF INTEREST}

Except for grant support noted above, the authors have no other association that may pose a conflict of interest, financial or otherwise.

\section{REFERENCES}

[1] Cortese, M.; Parashar, U.D. Prevention of rotavirus gastroenteritis among infants and children. Morb. Mortal. Wkly. Rep., 2009, 58 (RR-2), 1-24.

[2] Committee on Infectious Diseases. Prevention of rotavirus disease: updated guidelines for use of rotavirus vaccine. Pediatrics, 2009, 123 (5), 1412-1420.

[3] Grimwood, K.; Buttery, J.P. Clinical update: rotavirus gastroenteritis and its prevention. Lancet, 2007, 370, 302-304.

[4] Centers for Disease Control and Prevention. Delayed onset and dimished magnitude of rotavirus activity - United States, November 2007-May 2008. Morb. Mortal. Wkly. Rep., 2008, 57 (25), 697700 .

[5] Clark, H.F.; Lawley, D.; Mallette, L.A.; DiNubile, M.J.; Hodinka, R.L. Decline in cases of rotavirus gastroenteritis presenting to the Children's Hospital of Philadelphia after introduction of a pentavalent rotavirus vaccine. Clin. Vaccine Immunol., 2009, 16 (3), 382386.

[6] Estes, M.K.; Kapikian, A.Z. Field's Virology. Knipe, D.M.; Howley, P.M.; Eds. Rotaviruses. $5^{\text {th }}$ ed., Lippincott Williams and Williams, Philadelphia, PA: USA 2007, pp. 1917-1958.

[7] Clark, H.F.; Lawley, D.A.; Schaffer, A.; Patacsil, J.M.; Marcello, A.E.; Glass, R.I.; Jain, V.; Gentsch J. Assessment of the epidemic potential of a new strain of rotavirus associated with the novel G9 serotype which caused an outbreak in the United States for the first time in the 1995-1996 season. J. Clin. Microbiol., 2004, 42 (4), 1434-1438.

[8] Freeman, M.M.; Kerin, T.; Hull, J.; Teel, E.; Esona, M.; Parashar, U.; Glass, R.I.; Gentsch, J.R. Phylogenetic analysis of novel G12 rotaviruses in the United States: a molecular search for the origin of a new strain. J. Med. Virol., 2009, 81 (4), 736-746.

[9] Bégué, R.E.; Dennehy, P.H.; Huang, J.; Martin, P. Serotype variation of group A rotaviruses over nine winter epidemics in Southern New England. J. Clin. Microbiol., 1992, 30 (6), 15921594.

[10] Gentsch, J.; Gray, J.; Iturriza, M.; Klena, J. Manual of rotavirus detection and characterization methods. WHO Department of Vaccines and Biologicals: http://www.who.int/vaccines-documents/ (accessed June 3, 2009).

[11] Pavia, M.; Bianco, A.; Nobile, C.G.A.; Marinelli, P.; Angelillo, I.F. Efficacy of pneumococcal vaccination in children younger than 24 months: a meta-analysis. Pediatrics, 2009, 123 (6), e11031110 .

[12] Hicks, L.A.; Harrison, L.H.; Flannery, B.; Hadler, J.L.; Schaffneer, W.; Craig, A.S.; Jackson, D.; Thomas, A.; Beall, B. Lynfield, R.; Reingold, A.; Farley, M.M.; Whitney, C.G. Incidence of pneumococcal disease due to non-pneumococcal conjugate vaccine (PCV7) serotypes in the United States during the era of widespread PCV7 vaccination, 1998-2004. J. Infect. Dis., 2007, 196 (9), 13461354.

[13] Pichichero, M.E.; Casey, J.R. Emergence of a multiresistant serotype 19A pneumococcal strain not included in the 7-valent conjugate vaccine as an otopathogen in children. JAMA, 2007, 298 (15), 1772-1778

[14] Reingold, A.; Hadler, J.; Farley, M.M.; Harrison, L.; Lynfield, R.; Lexau, C.; Bennett, N.; Thomas, A.; Craig, A.S.; Smith, P.J.; Beall, B.; Whitney, C.G.; Moore, M.; Pilishvili, T. Invasive pneumococcal disease in children 5 years after conjugate vaccine introduction - Eight States, 1998-2005. Morb. Mortal. Wkly. Rep., 2008, 57 (6), 144-148.

[15] Gentsch, J.R.; Hull, J.J.; Teel, E.N.; Kerin, T.K.; Freeman, M.M.; Esona, M.D.; Griffin, D.D.; Bielfelt-Krall, B.P.; Banyai, K.; Jiang, B.; Cortese, M.M.; Glass, R.I.; Parashar, U.D. G and P types of circulating rotavirus strains in the United States during 1996-2005: nine years of prevaccine data. J. Infect. Dis., 2009, 200 (Suppl 1), S99-S105.

[16] Huang, J.; Wang, L.; Firth, S.; Phelps, A.; Reeves, P.; Holmes, I. Rotavirus VP7 epitope mapping using fragments of VP7 displayed on phages. Vaccine, 2000, 18 (21), 2257-2265.

[17] Taniguchi, K.; Hoshino, Y.; Nishikawa, K.; Green, K.Y.; Maloy, W.L.; Morita, Y.; Urasawa, S.; Kapikian, A.Z.; Chanock, R.M.; Gorziglia, M. Cross-reactive and serotype-specific neutralization epitopes on VP7 of human rotavirus: nucleotide sequence analysis 
of antigenic mutants selected with monoclonal antibodies. J. Virol., 1988, 62 (6), 1870-1874.

[18] Green, K.Y.; Kapikian, A.Z. Identification of VP7 epitopes associated with protection against human rotavirus illness or shedding in volunteers. J. Virol., 1992, 66 (1), 548-553.

[19] Franco, M.A.; Prieto, I.; Labbé, M.; Poncet, D.; Borras-Cuesta, F.; Cohen J. An immunodominant cytotoxic T cell epitope on the VP7 rotavirus protein overlaps the $\mathrm{H} 2$ signal peptide. J. Gen. Virol., 1993, 74 (12), 2579-2586.
[20] Hansen, G.; Mehnert, F.; Streckert, H.J.; Werchau, H. Monoclonal antipeptide antibodies recognize epitopes upon VP4 and VP7 of simian rotavirus SA11 in infected MA104 cells. Arch. Virol., 1992, $122(3-4), 281-291$.

[21] Zhou, Y.J.; Burns, J.W.; Morita, Y.; Tanaka, T.; Estes, M.K. Localization of rotavirus VP4 neutralization epitopes involved in antibody-induced conformational changes of virus structure. $J$. Virol., 1994, 68 (6), 3955-3964.

(C) Sanzone and Bégué; Licensee Bentham Open.

This is an open access article licensed under the terms of the Creative Commons Attribution Non-Commercial License (http://creativecommons.org/licenses/by$\mathrm{nc} / 3.0 /$ ) which permits unrestricted, non-commercial use, distribution and reproduction in any medium, provided the work is properly cited. 